\title{
UBV Observations of the Ultrashort Period Variable HZ 29
}

\author{
W. KRZEMINSKI (Warsaw)
}

The periodic light variability of $\mathrm{HZ} 29(=\mathrm{AM} \mathrm{CVn})$ discovered in 1962 by SMAK (1967) was subsequently studied in detail by OSTRIKER and HESSER (1968; hereinafter referenced as $\mathrm{OH}$ ). The observations of SMAK were confirmed to the blue and ultraviolet light and those of OSTRIKER and HESSER to the integrated light; these revealed light variations in the form of a slightly distorted double sinusoid. This distortion manifests itself that the alternate maxima are broader and, consequently, the alternate minima occur near phase $0.54 \mathrm{P}$ (when phases are counted from minima preceding broader maxima). The over-all amplitude of light oscillations is between 0.02 and 0.05 mag., the period, as determined by $O H$, is $1051.118 \pm 0.015 \mathrm{sec}$.

The peculiar spectrum of $\mathrm{HZ} 29$ was classified as a DBp (a hydrogen deficient white dwarf). It is characterized by very shallow, apparently double absorption lines of He I (GREENSTEIN and MATTHEWS 1957). Photoelectric scanner spectra studied by WAMPLER (1967) indicate that $\mathrm{HZ} 29$ is a hot star with a temperature of $\mathrm{T}_{e} \approx 20000^{\circ} \mathrm{K}$. In 1967 GREENSTEIN and KRAFT (private communication) attempted to detect radial velocity changes using single trailed spectra with a period of $1050 \mathrm{sec}$. or half that value and making multiple exposures. No evidence for radial velocity changes was found, however.

The photometric observations reported here were obtained in the first half of 1967 on the Mount Wilson telescopes. The purpose of these observations was to confirm the SMAK's discovery, to improve the period and to study the colour variations. The present observations were not reported earlier because of scarcity of the author's data and the subsequent publication of extensive investigation by OSTRIKER and HESSER (1968).

The author's photometric data consist of several short runs performed on eight nights; the 100-inch telescope was utilized to the UBV observations and the 60-inch to the blue measurements. A single beam conventional photoelectric photometer with offset guiding was used throughout in a pulse counting mode with $10 \mathrm{sec}$. counting intervals. The method of reduction and journal of observations will be given elsewhere (KRZEMINSKI 1972). Altogether 6 hours 30 minutes of observing time was devoted to the variable alone (excluding measurements of sky, comparison star, reset of digital counters, etc.). The mean error of a single measurement due to photon statistics is around $0.01 \mathrm{mag}$. for the average observation.

The individual runs very often exhibited large intrinsic scatter much in excess of errors of single measurements. In addition, the mean light level varied during the observing season. In order to study the general pattern of light variations, the individual runs were grouped into monthly mean light curves using the period given by $\mathrm{OH}$. Such mean light curves in the blue light are shown in Figure 1; the lengths of vertical bars are twice the formal standard error of the mean of all observations included in each point. Inspection of this figure reveals generally the same pattern which was found in much greater detail by $\mathrm{OH}$. In spite of the paucity of the data entering the mean curves, however, one may clearly see the double wave with the characteristic wider and narrower maxima.

One may also notice that the period given by $\mathrm{OH}$ does not fit all the observations; the phase shift indicates period to be shorter than that given by $\mathrm{OH}$; in addition one notes that the period derived from pairs of observing runs which include observations obtained in April 1967 differs slightly from that determined from all other pairs. It was known from observations of SMAK (1967) and OH that period determined from some pairs of runs differed considerably from its mean value (cf. Fig. 2 of $\mathrm{OH}$ ). Consequently, it was decided to form two groups of observations with slightly different values of period: the first included all UBV data (February - April 1967) while the second the observations obtained in the blue (June - July 1967). The preliminary values of periods were further refined by choosing that value of period which minimizes the dispersion about the mean light curve in either group. The mean UBV light and colour curves were constructed using elements: 
B

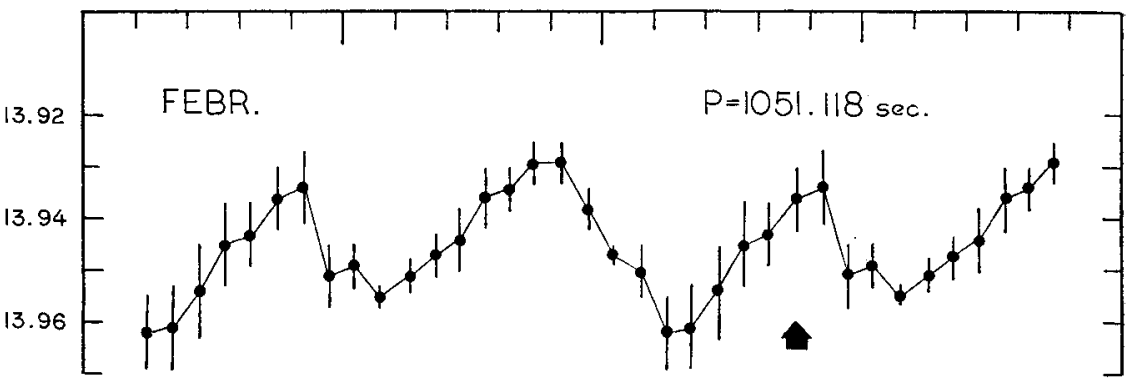

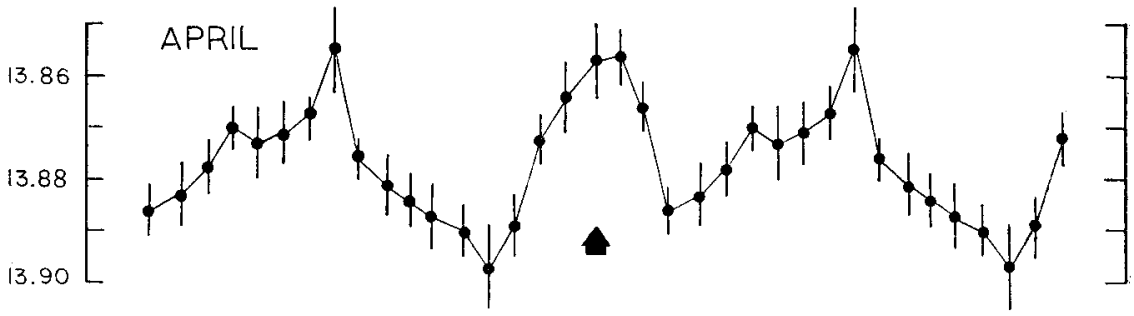
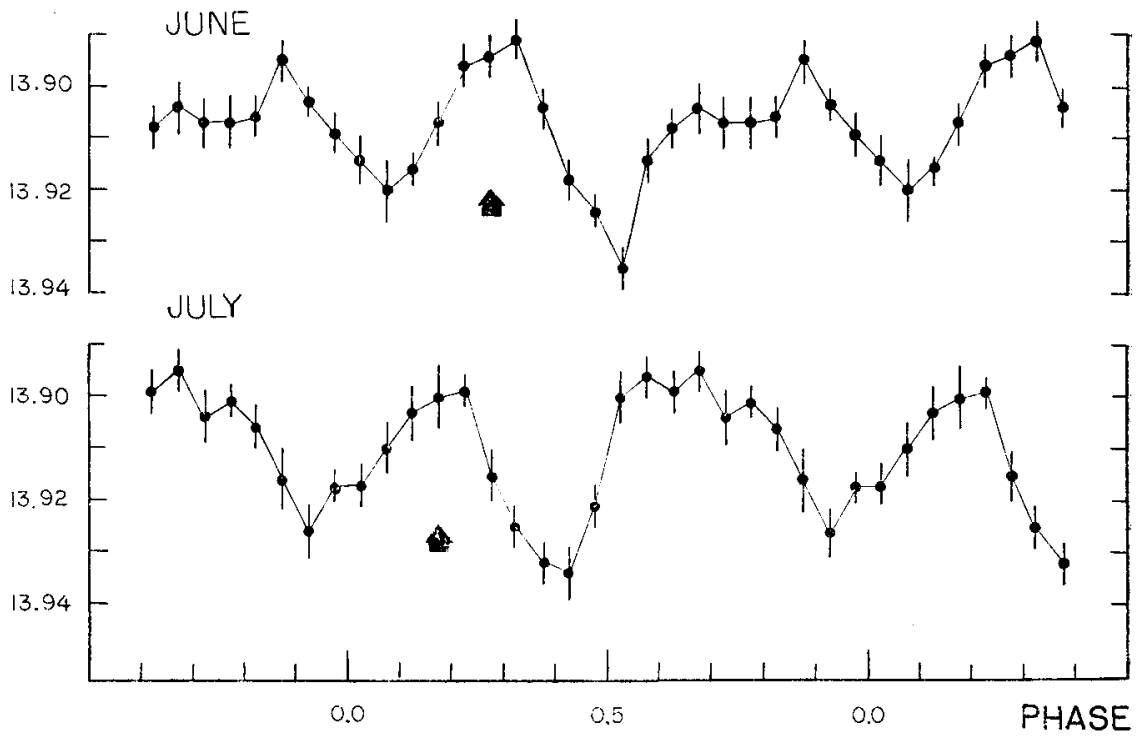

Fig. 1: Light curves of $\mathrm{HZ} 29$ in the blue light obtained in 1967. Zero phase is defined to occur at J. D. hel. 2439526.0 . Arrows indicate narrower maxima. 


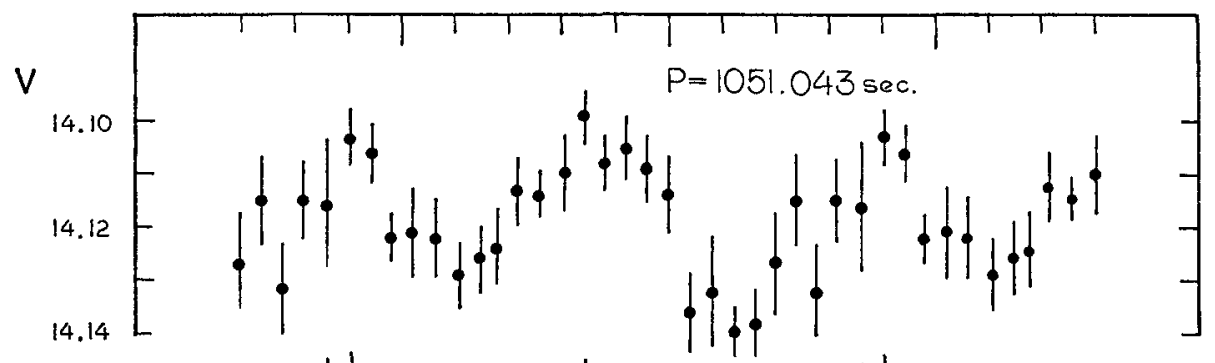

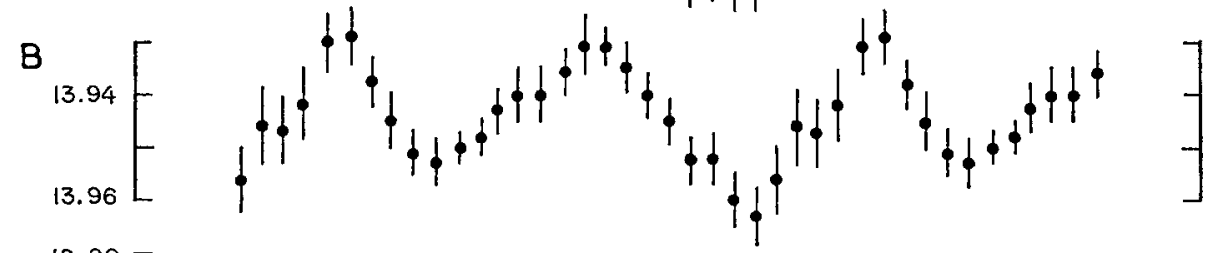

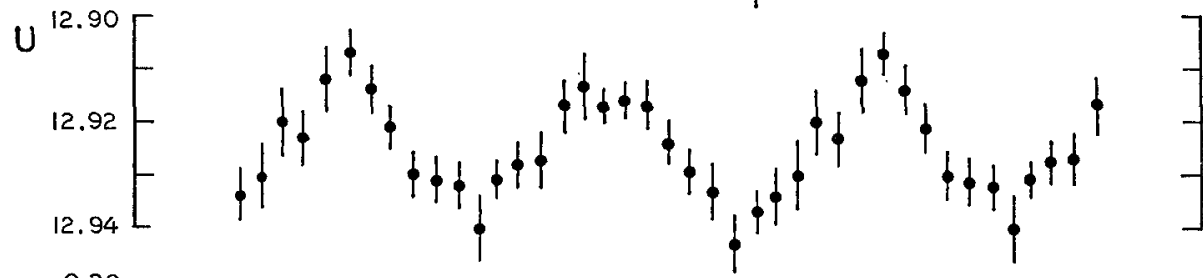

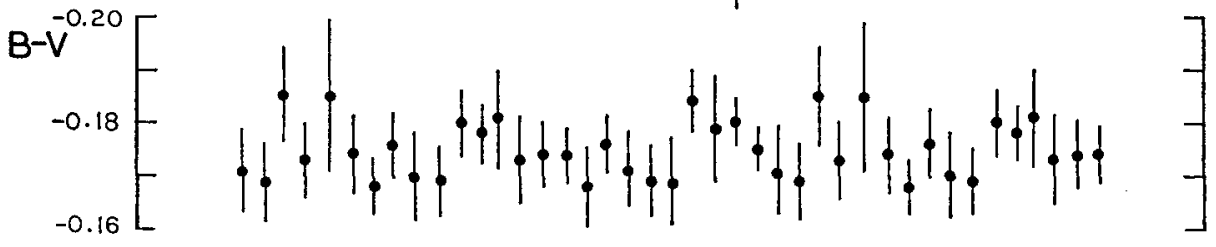

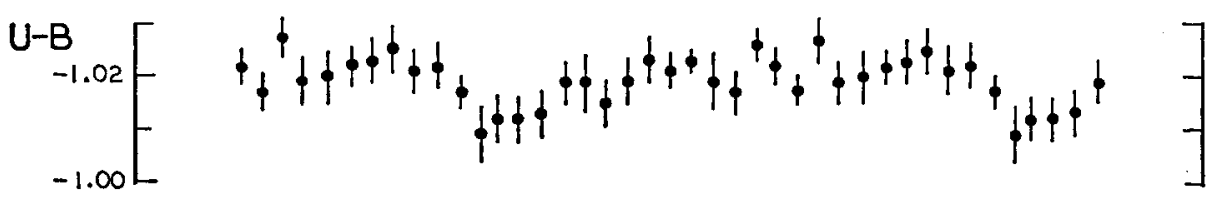

$P=1051.056$ sec.

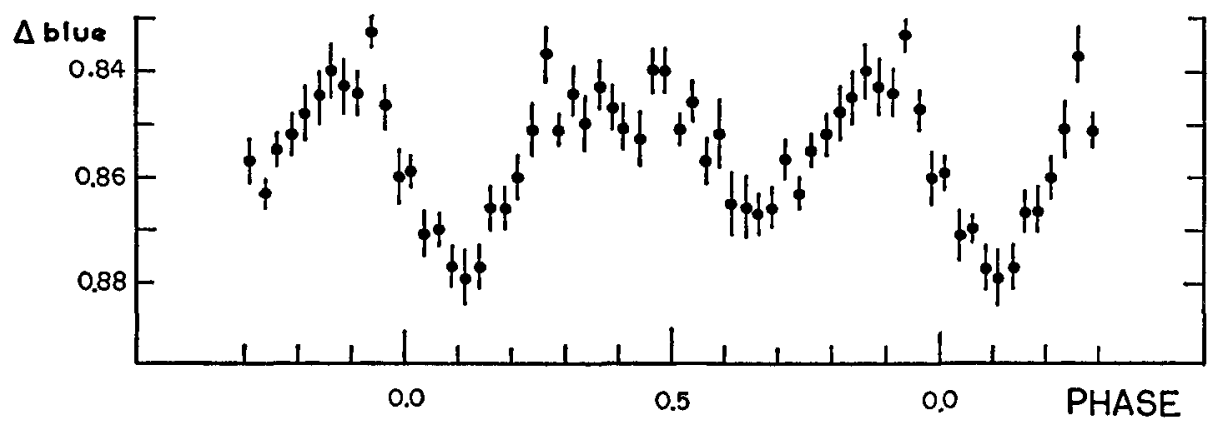

Fig. 2: Mean UBV light and colour curves of HZ 29 obtained in February and April 1967 and the blue light curve of June and July 1967. Phases are as in Fig. 1. 
Zero phase $=$ J. D. hel. $2439526.0+0.01216485 \times$ E (i. e. $P=1051.043$ sec.), while the mean blue light curve was constructed using the elements:

Zero phase $=$ J. D. hel. $2439526.0+0.01216500 \times$ E (i. e. $P=1051.056$ sec.) (cf. Figure 2) .

All the author's observations are displayed in Figure 2. One may note that during February - April 1967 the minimum preceding broader maximum (which, for convenience, is to be called the primary minimum) was shallower than the secondary minimum while during June - July 1967 the situation reversed. In this context it is worth noting that in 1962 (SMAK 1967) the secondary minimum was slightly shallower than the primary while in 1968 (cf. Fig. 3 of $\mathrm{OH}$ ) both minima were of the same depth. The B-V colour curve seems to be a mirror image of the light curves. It shows slight B-V excess during minima and a weak depression at light maxima (amounting to about 0.003 mag. at broader maximum). Similar effect, i. e. that star is reddest at maximum light was found by HESSER and LASKER (1971) for the periodically variable white dwarf $\mathrm{R} 548$. The $\mathrm{U}-\mathrm{B}$ colour curve shows depression at primary minimum and an excess at maxima; a weak depression at secondary minimum can barely be distinguished. Parenthetically one may note that the light and colour variations of $\mathrm{HZ} 29$ throughout the cycle resemble those of a small subgroup of magnetic variables with two light maxima (cf. STEPIEN 1968).

The physical interpretation of $\mathrm{HZ} 29$ is still a matter of controversial hypotheses (cf. $\mathrm{OH}$, Sec. IV). Any satisfactory model of this star should explain both the peculiar double wave light variations with accompanying colour changes and a small, presumably erratic period variation. Any such model, at the moment, is lacking. The general trend in interpretations of this object and other ultrashort-period white dwarfs goes towards the models which couple the rotation with some kind of oscillations (cf. LASKER and HESSER 1971).

$$
\text { References: }
$$

GREENSTEIN, J. L., and MATTHEWS, M. S., 1957, Ap. J., 126, 14.

HESSER, J. E., and LASIKER, B. M., 1971, Bull. Am. Astr. Soc., 3, 242.

KRTEMINSKI, W., 1972, Acta Astr., 22 (in preparation).

LASKER, B. M., and HESSER, I. E., 1971, Ap. I. (Letters), 163, L89.

OSTRIKER, J. P., and HESSER, J. E., 1968, Ap. J. (Letters), 153, L151.

SMAK, J., 1967, Acta Astr., 17, 255.

STEPIEN, K., 1968, Ap. J., 154, 945.

WAMPLER, E. J., 1967, Ap. J. (Letters), 149, L101.

\section{Discussion to the paper of KRZEMINSSKI:}

SMAK: I wish to add two comments:

1. My own observation made in 1962 could be reasonably well fitted with exactly the same period as that found later by OSTRIKER and HESSER, except for two runs which showed significant phase shifts.

2. It appears now from the observations by KRZEMINSKI, OSTRIKER and HESSER, WARNER and myself that the light curve of $\mathrm{HZ} 29$ is not stable; on many occasions the regular 18 -min. variations seem either absent or replaced by irregular noise. 